\title{
Urethral Carcinoma
}

National Cancer Institute

\section{Source}

National Cancer Institute. Urethral Carcinoma. NCI Thesaurus. Code C9106.

A carcinoma that arises from the urethra. Morphologically, the tumors are similar to those described in the bladder. 